\title{
Contribución de los Componentes de la Memoria de Trabajo a la Eficiencia en Aritmética Básica Durante la Edad Escolar
}

\section{Contribution of Working Memory Components to Efficiency in Simple Arithmetic During School Age}

\author{
Danilka Castro \\ Universidad de Chile
}

\author{
Valeska Amor \\ Centro de Neurociencias de Cuba
}

\author{
David Maximiliano Gómez y Pablo Dartnell \\ Universidad de Chile
}

\begin{abstract}
Aunque existen múltiples evidencias respecto de la relación entre la memoria de trabajo (MT) y el rendimiento aritmético, los resultados aún son inconsistentes. El objetivo del presente estudio fue evaluar la contribución única de cada componente de la MT (bucle fonológico, agenda visuo-espacial y ejecutivo central) a la explicación de la varianza de la eficiencia en aritmética básica, durante diferentes momentos del desarrollo escolar. Se evaluaron 285 estudiantes ( $M$ edad: 9,58 años, $D E=1,79$ ) de 5 establecimientos educacionales de Santiago y Chillán, Chile, con y sin dificultades en aritmética básica, a través de tareas de MT. Análisis de regresiones jerárquicas mostraron que los componentes de la MT tienen una contribución única a la varianza de la eficiencia en aritmética básica, pero esta varía durante el desarrollo escolar. Análisis de varianza mostraron diferencias en el funcionamiento de la MT entre estudiantes con y sin dificultades en aritmética, lo cual sugiere que el origen de estas dificultades podría estar muy asociado a la MT. Estos resultados tienen especial relevancia para explicar el desarrollo de la cognición numérica durante la edad escolar.
\end{abstract}

Palabras clave: memoria de trabajo, aritmética básica, habilidades numéricas, dificultades de aprendizaje

\begin{abstract}
Although several studies have provided evidence for the relation between working memory (WM) and arithmetic performance, results are still inconsistent. The aim of this study was to evaluate the unique contribution of each component of WM (phonological loop, visuo-spatial sketchpad and central executive) to the explained variance of efficiency in simple arithmetic during different moments of school-age development. WM tasks were used to evaluate 285 students $(M$ age $=9.58$ years, $S D=1.79)$, with and without difficulties in simple arithmetic, from 5 schools in Santiago and Chillán, Chile. Hierarchical regression analyses showed that each WM component contributes differently to the variance of efficiency in simple arithmetic; however, this contribution varies throughout students' school-age development. Variance analysis showed differences in WM functioning between students with and without difficulties in arithmetic, which suggests that the origin of such difficulties may be strongly linked to WM. These results are especially relevant for explaining the development of numerical cognition during school age years.
\end{abstract}

Keywords: working memory, simple arithmetic, numeracy skills, learning difficulties

La habilidad para realizar operaciones aritméticas es una de las destrezas académicas primarias que los niños aprenden en la escuela y que luego acompaña el desempeño en la sociedad. Las teorías recientes sobre el desarrollo de competencias matemáticas consideran que las capacidades numéricas básicas (e.g., conteo, comparación, estimación) constituyen el andamiaje cognitivo sobre el cual se construyen las destrezas aritméticas de más alto nivel (Feigenson, Dehaene \& Spelke, 2004). Sin embargo, varios estudios han revelado que durante la edad escolar existe, además, una relación causal entre los procesos de dominio general - particularmente de los componentes de la memoria de trabajo (MT) - y las habilidades aritméticas, tanto durante el desarrollo típico como atípico de la cognición numérica (Bull \& Johnston, 1997; Fuchs et al., 2010; Geary, 2011; Swanson, 2011). A pesar del creciente número de evidencias respecto de la interacción

Danilka Castro Cañizares, David Maximiliano Gómez y Pablo Dartnell, Laboratorio de Neurociencia y Cognición, Centro de Investigación Avanzada en Educación, Universidad de Chile, Santiago, Chile; Valeska Amor Díaz, Departamento de Neurociencias Educacionales, Centro de Neurociencias de Cuba, La Habana, Cuba.

Este proyecto fue realizado gracias al financiamiento otorgado por el Proyecto Basal FB0003 del Programa de Investigación Asociativa de la Comisión Nacional de Investigación Científica y Tecnológica.

La correspondencia relativa a este artículo debe ser dirigida a Danilka Castro Cañizares, Centro de Investigación Avanzada en Educación, Universidad de Chile, Periodista José Carrasco Tapia 75, 8330014, Santiago, Chile. E-mail: danilka.castro@ciae.uchile.cl 
entre el desempeño aritmético (y matemático en general) y la MT, esta no ha sido considerada como componente en las principales teorías de la cognición numérica, por ejemplo, en el modelo de código abstracto (McCloskey, 1992) o en el modelo del triple código (Dehaene, 1992). Por tanto, estos modelos pudieran estar perdiendo un importante constructo explicativo para entender tanto el funcionamiento como el desarrollo de las habilidades aritméticas.

Siguiendo el modelo multi-componentes propuesto por Baddeley y Hitch (1974), la MT se considera como un sistema cognitivo central de capacidad limitada, encargado de manipular y almacenar temporalmente la información necesaria para realizar tareas complejas, como la comprensión del lenguaje o el razonamiento. En este modelo, la MT se divide en tres componentes: un ejecutivo central y dos sistemas pasivos de almacenamiento temporal de información, el bucle fonológico y la agenda visuo-espacial.

El componente ejecutivo central se encarga de coordinar el intercambio de información con los componentes de memoria a corto y largo plazo y no tiene modalidad específica de procesamiento. También es el responsable de los procesos de asignación de los recursos atencionales (división atencional en doble tarea, focalización, inhibición de distractores), las operaciones de evaluación y monitoreo de información, el cambio de actividad cognitiva y la actualización de contenidos, y de la recuperación estratégica de información desde la memoria a largo plazo (Baddeley, 1996, 2003; Baddeley \& Hitch, 1994). Considerando que las tareas aritméticas (y numéricas en general), dada su naturaleza, requieren de la actualización y manipulación de información cuantitativa, verbal y espacial, es esperable que al menos cierto desarrollo de las habilidades de MT sea requerido para el desempeño exitoso en este tipo de tareas.

El bucle fonológico almacena información de tipo verbal y auditiva. Este sistema auxiliar se compone de un almacén fonológico, que procesa y retiene la información acústica-verbal durante unos segundos, y de un mecanismo de repetición sub-vocálico que fortalece la huella de la información. Por tanto, el bucle fonológico se encuentra involucrado en la comprensión y adquisición del lenguaje y está especialmente implicado en el aprendizaje de la lecto-escritura (Baddeley, 1992). Respecto de las matemáticas, su rol primario es codificar y retener los códigos verbales necesarios para el conteo y se vincula con la recuperación de los hechos numéricos, la aritmética exacta y los algoritmos matemáticos, como la adición y la sustracción (Fuchs et al., 2006).

Por su parte, la agenda visuo-espacial es la responsable de la elaboración y manipulación de la información visual y espacial implicada en tareas que suponen memoria espacial. Es responsable de generar y manipular imágenes mentales. Se ha sugerido que la agenda visuo-espacial está fraccionada en dos componentes: uno visual y otro espacial, de modo que las interferencias espaciales que no requieren procesamiento visual llevan a una disminución del rendimiento en tareas de seguimiento de secuencias en movimiento, pero no en el recuerdo de imágenes estáticas o colores (Logie, 1995; Quinn \& McConnell, 1999). En el caso de las matemáticas, la agenda visuo-espacial está involucrada en el seguimiento de secuencias aritméticas, el conteo y la comparación de cantidades (De Smedt, Verschaffel \& Ghesquière, 2009). Se ha descrito que las magnitudes numéricas se representan en forma de una línea numérica mental (Dehaene, Piazza, Pinel \& Cohen, 2003). Por tanto, un déficit en la manipulación y mantenimiento de las representaciones espaciales podría también estar afectando el sentido de la magnitud numérica.

Respecto de la contribución única de cada uno de estos componentes en el rendimiento matemático, los resultados son diversos. Algunos autores encuentran que solo la MT visuo-espacial está vinculada al desempeño en matemáticas, pero no la MT verbal (Amor Díaz \& Torres Díaz, 2016; Dumontheil \& Klingberg, 2012), mientras que otros reportan que las tareas de MT verbal se vinculan al desempeño matemático solo si involucran números (Wu et al., 2008). A pesar de lo anterior, Friso-van den Bos, van der Ven, Kroesbergen y van Luit (2013), en su meta-análisis sobre estudios que exploran la relación entre el desempeño matemático y los componentes de la MT en niños de entre 4 y 12 años de edad, mostraron que, de manera general, todos estos componentes aportan una contribución única a la varianza en el desempeño matemático, independientemente de otras variables de dominio general, como la inteligencia, la habilidad lectora, la edad y la memoria a corto plazo.

Otro grupo de evidencias respecto de la contribución de la MT a la eficiencia en aritmética proviene de estudios con niños que presentan dificultades en el aprendizaje de las matemáticas. Se ha descrito que los niños con dificultades en este dominio son menos propensos a usar recuperaciones directas de hechos numéricos desde la memoria a largo plazo para resolver tareas aritméticas y sus estrategias de conteo son mucho más lentas e imprecisas que las usadas por los niños sin dificultad (Bull \& Johnston, 1997). Además, estos niños frecuentemente obtienen menores puntajes en mediciones del bucle fonológico (Bull \& Johnston, 1997; Passolunghi \& Siegel, 2001), el cual se ha descrito que apoya la recuperación de hechos numéricos 
desde la memoria a largo plazo (Dehaene \& Cohen, 1995), y muestran un desempeño por debajo de la norma en mediciones de la agenda visuo-espacial (McLean \& Hitch, 1999). Los resultados de dos meta-análisis realizados previamente (David, 2012; Swanson \& Jerman, 2006) dan cuenta que los niños con dificultades específicas para las matemáticas también tienen un menor rendimiento en tareas que evalúan el componente ejecutivo central de la MT, especialmente cuando se involucra información numérica. En un estudio realizado por Szucs, Devine, Soltesz, Nobes y Gabriel (2013) con niños de entre 9 y 10 años (12 con discalculia y 12 controles) sobre las causas que provocan la discalculia del desarrollo, los autores cuestionan la teoría del déficit en la representación de las cantidades como causa originaria de este trastorno del aprendizaje. A partir del análisis de sus datos y de las evidencias previas, señalan que la disfunción más robusta para explicar el origen de la discalculia del desarrollo es un pobre funcionamiento de la MT, derivado de dificultades en la memoria a corto plazo, y de una disfunción en la MT visuo-espacial y en la función de inhibición (supresión de interferencias), ambas ligadas al funcionamiento del ejecutivo central en la MT.

Como se ha descrito anteriormente, los hallazgos respecto a qué componentes de la MT pueden realmente predecir los desempeños matemáticos y cuáles no son aún inconsistentes. La mayoría de los estudios previos utiliza como variable dependiente los resultados de pruebas institucionalizadas basadas en el currículo, en las que se incluyen múltiples dominios matemáticos y no hay control del tiempo de respuestas (Kyttälä \& Lehto, 2008; Passolunghi \& Cornoldi, 2008). Sin embargo, la fluidez en el cálculo y el rendimiento curricular podrían estar representando distintos dominios de la cognición numérica. Por ejemplo, se ha descrito que los niños con un rendimiento inferior a la norma en tareas de cálculo tienen perfiles cognitivos diferentes, comparados con aquellos que presentan menor rendimiento en tareas de resolución de problemas (Fuchs et al., 2010; Träff, 2013). Asimismo, se ha señalado que los niños con dificultades específicas en las matemáticas podrían resolver tareas numéricas usando estrategias ineficientes que requieren de mayor tiempo para obtener la solución correcta (Jordan \& Montani, 1997). De esta forma, en su rendimiento se involucran procesos de MT que no son evaluados en las pruebas curriculares. Además, varias evidencias muestran que la aritmética simple y otras habilidades numéricas básicas como, por ejemplo, las habilidades de procesamiento de símbolos numéricos y las vinculadas al sistema numérico aproximado, son buenos predictores del desempeño posterior en tareas matemáticas (De Smedt et al., 2009; LeFevre et al., 2010; Reigosa-Crespo et al., 2013). Considerando estos elementos, para explorar la contribución que tienen los componentes de la MT al desempeño matemático, resultaría recomendable el uso de tareas que exploren el rendimiento aritmético básico con control del tiempo de reacción (TR).

Otra causa probable de la inconsistencia entre las evidencias previas surge del hecho de que la contribución de cada componente de MT podría variar en función de la edad y del nivel alcanzado por los niños en el aprendizaje de las matemáticas. Sin embargo, pocas investigaciones se han dirigido a evaluar esta variable (Cragg \& Gilmore, 2014). Los resultados de un estudio realizado por Berg (2008) con niños de entre 8 y 12 años mostraron que la edad tiene una contribución al cálculo aritmético, aun cuando se controlan otras variables. Al respecto, se ha descrito que la agenda visuo-espacial tiene un rol independiente del resto de los componentes de la MT en los primeros años de aprendizaje de las matemáticas. McKenzie, Bull y Gray (2003) encontraron que alrededor de los 6-7 años el desempeño aritmético se afectaba solo por dificultades en la MT visuo-espacial, mientras que a los 8-9 años este desempeño se afectaba tanto por dificultades verbales como visuo-espaciales de la MT. De manera similar, Holmes y Adams (2006) encontraron que la agenda visuoespacial predice una varianza única de las habilidades de rendimiento matemático en los niños pequeños (7-8 años). Sin embargo, en niños mayores (9-10 años) este componente de la MT solo fue predictor del rendimiento en los problemas aritméticos de mayor complejidad, lo cual pudiera reflejar una dependencia o una regresión a las estrategias visuo-espaciales cuando la aritmética simbólico-lingüística (Houdé, 1997) o las estrategias directas de recuperación de información no pueden ser aplicadas (Holmes \& Adams, 2006). Alrededor de los 9-10 años los niños comienzan ya a utilizar el bucle fonológico para la solución de problemas matemáticos sencillos, mostrando así, el dominio de la aritmética simbólico-lingüística o el uso de estrategias de solución más maduras (como la recuperación directa desde la memoria) que se basan en un código verbal (Berg, 2008; Dehaene \& Cohen, 1995; Houdé, 1997). Lo anterior lleva a considerar que algunas de las diferencias encontradas entre los estudios respecto de la influencia de los componentes de la MT en el desempeño aritmético pudieran deberse solo a que los participantes se encuentran en diferentes etapas de su desarrollo.

En último lugar, se podría destacar que en los estudios previos que vinculan a la MT con un desempeño aritmético en particular se controlan usualmente habilidades de dominio general, como la inteligencia, la velocidad de procesamiento, las habilidades de lectura y las habilidades fonológicas (Alloway et al., 2005; 
Amor Díaz \& Torres Díaz, 2016). Sin embargo, dada la influencia que tienen las habilidades numéricas básicas (como la comparación de cantidades) sobre el desempeño aritmético posterior (Hyde, Khanum \& Spelke, 2014; Reigosa-Crespo et al., 2013), resultaría de interés explorar cuál es realmente la contribución única que aporta la MT a la varianza del rendimiento aritmético cuando también se controla la contribución de las variables de dominio específico (variables de procesamiento numérico básico).

En concordancia con lo anterior, el presente estudio tuvo como objetivo evaluar la contribución única que aportan los componentes de la MT (bucle fonológico, agenda visuo-espacial y ejecutivo central) a la explicación de la varianza de la eficiencia en aritmética básica, en diferentes momentos del desarrollo escolar (desde $1^{\circ}$ hasta $6^{\circ}$ grado de enseñanza primaria). Para ello se evaluaron niños sin dificultades (grupo SDA) y con dificultades (grupo CDA) en el rendimiento aritmético, a través de tareas en las que se involucran los diferentes componentes de la MT. Para explorar la contribución única de estos componentes a la explicación de dicha varianza, en los análisis realizados se controlaron variables de dominio general (edad e inteligencia) y variables de dominio numérico que se ha descrito influyen en el rendimiento aritmético (eficiencia en la comparación de magnitudes simbólicas y no-simbólicas). Se esperaba que la MT (o al menos alguno de sus componentes) mostrara una contribución única a la explicación de la varianza en la eficiencia en aritmética básica en el grupo de niños SDA. Además, se esperaba que esta contribución fuera diferente en cada grupo etario evaluado, producto tanto del continuo desarrollo del sistema numérico aproximado como del de sarrollo de habilidades en la manipulación de los símbolos numéricos. Por otra parte, si las dificultades en aritmética básica (en el grupo de niños CDA) se deben solo a variables de dominio específico (habilidades numéricas básicas simbólicas y no-simbólicas), se esperaba que los resultados de ambos grupos (SDA y CDA) fueran similares tanto en el rendimiento en las tareas de MT como en la contribución única de cada uno de los componentes de MT a la eficiencia en la aritmética. Por el contrario, si la MT (o alguno de sus componentes) tiene una contribución única a la eficiencia en aritmética básica, podía esperarse que los niños del grupo CDA mostraran un rendimiento significativamente menor que el grupo SDA en estas tareas, al menos en aquellos componentes con contribución a la eficiencia en la aritmética.

\section{Método}

\section{Participantes}

Para la selección de los participantes se utilizó un muestreo de tipo no probabilístico por conveniencia. Se contactó a los directores de siete establecimientos educacionales de las ciudades de Santiago y Chillán, Chile. La selección inicial de los niños se realizó a partir de las respuestas de los profesores a un cuestionario de signos de riesgo de presentar dificultades en el aprendizaje matemático. Los profesores completaron un cuestionario por cada niño de su clase, de modo que el profesor ofreció información sobre todos los sujetos y no estuvo involucrado en la selección de estos. Los niños sin ningún signo de riesgo en este cuestionario fueron incluidos en el grupo SDA. Aquellos niños con al menos un signo de riesgo de trastornos en el aprendizaje fueron incluidos en la primera selección de niños CDA

Posteriormente, todos los niños realizaron el Test de Matrices Progresivas Coloreadas de Raven (Raven, Court \& Raven, 1992) que evalúa inteligencia no verbal. Para ser incluidos en la muestra, los niños debían obtener puntajes que los ubicaran entre los percentiles 50 y 95 en este test. Finalmente, los participantes seleccionados fueron evaluados a través de un test de aritmética básica (ver descripción más adelante). Para la inclusión definitiva en el grupo CDA se utilizó el Test de Crawford (Crawford \& Howell, 1998). Este procedimiento posibilita hacer comparaciones precisas con tamaños de muestra $(N)$ más modestos, sin correr el riesgo de exagerar la anormalidad de las puntuaciones, como ocurre con el valor $Z$, cuando se emplean muestras pequeñas. Siguiendo este método, se incluyeron en el grupo CDA a aquellos sujetos cuya eficiencia en el test de aritmética básica estuvo dos desviaciones estándar por debajo de la media de la eficiencia del grupo SDA.

La muestra final quedó conformada por un total de 285 niños de 30 salas de clases pertenecientes a cinco establecimientos educacionales de enseñanza primaria, dos de la ciudad de Santiago y tres de la ciudad de Chillán. De ellos, 225 ( $M$ edad: 9,53 años, $D E$ : 1,79) conformaron el grupo de niños SDA. Este grupo se dividió en tres subgrupos, atendiendo al grado escolar de los participantes: un subgrupo de niños que se encontraba en las fases iniciales de la enseñanza formal de las matemáticas (subgrupo 1: niños de $1^{\circ}$ y $2^{\circ}$ grado escolar), un subgrupo intermedio (subgrupo 2: niños de $3^{\circ}$ y $4^{\circ}$ grado escolar) y un subgrupo de niños con mayor tiempo de exposición a la enseñanza de las matemáticas (subgrupo 3: niños de $5^{\circ}$ y $6^{\circ}$ grado escolar). Sesenta sujetos 
( $M$ edad: 9,79 años, $D E$ : 1,77) conformaron el grupo de niños CDA, el cual fue dividido en tres subgrupos, al igual que el grupo de niños SDA. En la Tabla 1 se muestran los detalles de cada subgrupo.

Tabla 1

Detalles de la Muestra

\begin{tabular}{lcccccccc}
\hline & \multicolumn{4}{c}{ Grupo SDA } & \multicolumn{4}{c}{ Grupo CDA } \\
\cline { 2 - 9 } $\begin{array}{c}\text { Grado de } \\
\text { enseñanza }\end{array}$ & $N$ & $\begin{array}{c}\text { Span de } \\
\text { dígitos } \\
\text { progresivo }\end{array}$ & $\begin{array}{c}\text { Span de } \\
\text { dígitos } \\
\text { regresivo }\end{array}$ & $\begin{array}{c}\text { Span } \\
\text { visuo- } \\
\text { espacial }\end{array}$ & $N$ & $\begin{array}{c}\text { Edad } \\
(D E)\end{array}$ & $\begin{array}{c}\text { CI } \\
(D E)\end{array}$ & $\begin{array}{c}\text { Eficiencia } \\
\text { aritmética } \\
\text { básica }(D E)\end{array}$ \\
\hline Subgrupo 1 & 75 & 7,48 & 85,4 & 6991,46 & 20 & 7,83 & 72,7 & 14033,47 \\
& & $(0,65)$ & $(12,57)$ & $(2207,88)$ & & $(0,67)$ & $(18,38)$ & $(2900,64)$ \\
Subgrupo 2 & 75 & 9,59 & 70,4 & 3880,48 & 20 & 9,85 & 67,0 & 6213,20 \\
& & $(0,69)$ & $(16,78)$ & $(1213,60)$ & & $(0,82)$ & $(16,89)$ & $(1867,91)$ \\
Subgrupo 3 & 75 & 11,51 & 64,2 & 2916,37 & 20 & 11,71 & 63,5 & 7393,56 \\
& & $(0,78)$ & $(18,05)$ & $(872,10)$ & & $(0,82)$ & $(19,20)$ & $(2694,75)$ \\
\hline
\end{tabular}

\section{Instrumentos}

\section{Tareas de memoria de trabajo.}

Span de dígitos. Se utilizó el sub-test de la Escala de Inteligencia para Niños de Wechsler - Revisado (Sattler, 1982). La versión progresiva del Span de Dígitos fue aplicada como medición del componente bucle fonológico de la MT. Se ha descrito que las tareas de memoria a corto plazo, como el span de dígitos progresivo, solo requieren del mantenimiento de información (Cornoldi, Rigoni, Venneri \& Vecchi, 2000; Vecchi \& Cornoldi, 1999). La versión regresiva fue aplicada como medición del ejecutivo central durante la manipulación de información verbal. En las tareas que evalúan memoria de trabajo, como es el caso del span de dígitos regresivo, se realiza almacenamiento de información en un contexto donde se requiere procesamiento activo, el cual involucra el rol principal del componente ejecutivo central (Passolunghi \& Siegel, 2001, 2004).

Span visuo-espacial. Para explorar los componentes visuo-espaciales de la MT fueron diseñadas dos versiones (progresiva y regresiva) de un span visuo-espacial computarizado. De esta forma, el span visuoespacial fue equivalente al test de Span de Dígitos: la versión progresiva fue utilizada para explorar la agenda visuo-espacial y la versión regresiva, para explorar el ejecutivo central durante la manipulación de información visuo-espacial.

El diseño de la tarea fue el siguiente: Se presentó en la pantalla del computador, sobre fondo blanco, una grilla con 20 cuadrados. Cada ensayo comenzó con la presentación de una secuencia de cuadrados rojos (una secuencia de cuadrados de la grilla fue cambiando su color de blanco a rojo a razón de un cambio cada $300 \mathrm{~ms}$ ). Terminada la secuencia, el color de fondo cambió de blanco hacia rosado y los niños debían responder haciendo clic con el mouse en las posiciones de la grilla que cambiaron de blanco a rojo, en el mismo orden en que cambiaron de color (versión progresiva) o en orden inverso a la secuencia presentada (versión regresiva). La tarea estuvo conformada por un total de 14 secuencias $(2,3,4,5,6,7$ u 8 estímulos, cada numerosidad se repitió dos veces). Antes del inicio de la tarea se presentaron cuatro ensayos para practicar. Se obtuvo un punto por cada par consecutivo de estímulos recordados en orden correcto. De esta forma, en un ensayo con cinco estímulos se podían obtener como máximo cuatro puntos. El puntaje total de la tarea fue calculado como la suma de los 14 ensayos (ver Tillman, Nyberg \& Bohlin, 2008, para un procedimiento similar).

\section{Tareas numéricas.}

Test de aritmética básica. Test computarizado con control del TR, con 56 ensayos presentados en dos bloques de operaciones aritméticas con números de un solo dígito (1-9): 28 adiciones y 28 sustracciones. Los estímulos fueron dígitos arábigos blancos presentados sobre fondo negro, en letra Arial con fuente 60. Se 
presentaron en la forma " $2+4$ ". Debajo de estos se presentaron simultáneamente dos alternativas de respuesta: una correcta y una incorrecta. Cada ensayo comenzó con la presentación del estímulo, el cual se mantuvo en pantalla hasta que el sujeto ofreció la respuesta. Los niños debían seleccionar la respuesta correcta lo más rápido posible, pero sin cometer errores. La respuesta estuvo seguida por un intervalo interestímulos de $500 \mathrm{~ms}$ (fondo negro). Al inicio de la tarea se presentaron seis ensayos para practicar.

Tareas de comparación numérica. Cada tarea de comparación (no-simbólica y simbólica) consistió en la presentación de 60 pares de numerosidades a comparar (numerosidades del 1-9, excepto el 5). Los ensayos fueron presentados en dos bloques de 30 estímulos cada uno. Cada ensayo comenzó con la presentación de un par de numerosidades que se mantuvo en pantalla hasta que se ofreció la respuesta, la cual estuvo seguida por un intervalo inter-estímulos de $500 \mathrm{~ms}$ (fondo negro). Al inicio de la tarea se presentaron seis ensayos para practicar.

Comparación no-simbólica. Se presentaron simultáneamente dos cuadrados blancos (lado = $55 \mathrm{~mm}$ ), los cuales contenían un número variable de puntos negros. Los niños debían seleccionar el cuadrado con mayor número de elementos (o menor, según la instrucción) lo más rápido posible, pero sin cometer errores. Estos cuadrados se presentaron sobre fondo negro y separados por un punto de fijación central de color rojo (distancia entre los cuadrados $=8 \mathrm{~mm}$ ). Para evitar que los niños usaran estrategias de respuestas basadas en variables perceptuales fueron generados tres sets de conjuntos: set con control de la densidad (se mantuvieron constantes la densidad del conjunto y el tamaño de los elementos), set con control de la superficie (se mantuvieron constantes el área total ocupada y la suma del área de los elementos) y set con control del área (se mantuvieron constantes el área total ocupada y el tamaño de los elementos del conjunto).

Comparación simbólica. Se presentaron simultáneamente dos dígitos arábigos blancos sobre fondo negro, en letra Arial con fuente 60. Los niños debían seleccionar el dígito de mayor magnitud numérica (o menor, según la instrucción) lo más rápido posible, pero sin cometer errores.

\section{Procedimiento}

El presente estudio se desarrolló siguiendo los principios éticos vigentes para la investigación con seres humanos. En aquellos establecimientos donde se obtuvo autorización para desarrollar el estudio se realizó una reunión explicativa con los directores y profesores, en la que se solicitó su consentimiento informado para su participación. Posteriormente, con igual procedimiento, se solicitó el consentimiento informado de los apoderados de los participantes. En último lugar, se realizó una reunión con los participantes, en la que se les explicó en qué consistía su participación y se solicitó su asentimiento para participar en el estudio.

Las evaluaciones se realizaron en una habitación tranquila dentro de la escuela. Las tareas experimentales fueron aplicadas en dos sesiones de 30-40 minutos cada una por los propios investigadores del estudio y por asistentes de investigación previamente entrenados en la aplicación de estas tareas. En la primera sesión fue administrado el Test de Matrices Progresivas de Raven y seguidamente el test de aritmética básica. En la segunda sesión fueron administradas las tareas de MT y de comparación numérica, presentadas en orden contrabalanceado.

\section{Análisis de Datos}

El rendimiento en las tareas numéricas fue calculado utilizando una medida de eficiencia (ME). Esta medida se obtiene dividiendo la mediana del TR (solo de las respuestas correctas) entre la proporción de respuestas correctas en la tarea $(\mathrm{ME}=\mathrm{TR} /$ proporción de respuestas correctas). La ME es una medida inversa: un mayor valor de esta medida refleja una menor eficiencia del sujeto en la tarea.

En primer lugar, para cada subgrupo de niños SDA y CDA, se realizaron análisis de correlación lineal de Pearson, con el fin de evaluar la posible relación entre la eficiencia en aritmética básica y las variables de dominio general (edad e inteligencia), las variables de dominio específico numérico (eficiencia en comparación no-simbólica y simbólica) y los componentes de la MT (el puntaje obtenido en span de dígitos progresivo y regresivo, span visuo-espacial progresivo y regresivo). Cuando existen más de dos variables que correlacionan significativamente entre sí, el análisis de correlación solo permite inferir que entre dichas variables existe varianza compartida, pero no identificar las influencias reales que cada una de estas variables tiene sobre el resto. Por tanto, para explorar la contribución específica de cada variable a la varianza de la variable 
dependiente (en este caso, la eficiencia en aritmética básica) es necesario realizar regresiones jerárquicas. La regresión jerárquica es un método altamente conservador desde el punto de vista matemático, ya que permite evaluar la contribución única de una variable o predictor específico, controlando el efecto de otras variables que se encuentran relacionadas con él y con la variable dependiente.

Considerando lo anterior, se realizaron regresiones jerárquicas para identificar la contribución única de cada componente de la MT a la explicación de la varianza de la eficiencia en aritmética básica en cada subgrupo de niños SDA y CDA. Las regresiones incluyeron tantos modelos como componentes de MT que se correlacionaron con la eficiencia en aritmética básica en el subgrupo de niños correspondiente. En cada modelo de regresión jerárquica se incluyeron como variables de control en el bloque 1 las variables de dominio general (edad e inteligencia), las variables de dominio específico (eficiencia en comparación simbólica y no simbólica) y el puntaje obtenido en las tareas que exploraron cada componente de la MT, excepto del componente de MT de interés en el modelo, el cual se incluyó por separado en el bloque 2. Cada una de estas variables fue incluida en el modelo solo si se correlacionó con la eficiencia en aritmética básica en el subgrupo de niños correspondiente.

En último lugar, para obtener mayor información sobre posibles diferencias en el desarrollo de los componentes de la MT entre los grupos estudiados, se realizaron análisis de varianza (ANOVA) de medidas repetidas con los resultados de los grupos de niños SDA y CDA en las tareas de MT verbal y visuo-espacial. Los análisis se realizaron para cada subgrupo por separado.

\section{Resultados}

\section{Correlaciones entre la Eficiencia en Aritmética Básica y las Variables de Dominio General y Específico}

El análisis de correlación entre la eficiencia en aritmética básica y las variables de dominio general, las variables de dominio específico numérico y los componentes de la MT en los diferentes subgrupos de niños de SDA y CDA, mostró que las variables se correlacionan de manera diferente en cada momento del desarrollo escolar. Los detalles se muestran en la Tabla 2. Aquí es importante recordar que la medida de eficiencia utilizada en este estudio es una medida inversa (un mayor valor expresa menor eficiencia), por tanto, es esperable que las correlaciones entre las tareas numéricas sean directas, mientras que las correlaciones entre la eficiencia en aritmética básica y las tareas de MT sean inversas.

Tabla 2

Correlaciones Lineales (r) Entre la Eficiencia en Aritmética Básica y la Edad y el Rendimiento en Tareas de Dominio General y Dominio Específico

\begin{tabular}{|c|c|c|c|c|c|c|c|c|}
\hline $\begin{array}{l}\text { Grupo y } \\
\text { subgrupo }\end{array}$ & Edad & $\begin{array}{l}\text { Puntaje } \\
\text { Raven }\end{array}$ & $\begin{array}{c}\text { Span de } \\
\text { dígitos } \\
\text { progresivo }\end{array}$ & $\begin{array}{l}\text { Span de } \\
\text { dígitos } \\
\text { regresivo }\end{array}$ & $\begin{array}{c}\text { Span } \\
\text { visuo- } \\
\text { espacial } \\
\text { progresivo }\end{array}$ & $\begin{array}{c}\text { Span } \\
\text { visuo- } \\
\text { espacial } \\
\text { regresivo }\end{array}$ & $\begin{array}{l}\text { Comparación } \\
\text { no-simbólica }\end{array}$ & $\begin{array}{c}\text { Comparación } \\
\text { simbólica }\end{array}$ \\
\hline \multicolumn{9}{|l|}{ Grupo SDA } \\
\hline Subgrupo 1 & $-0,41^{* * *}$ & $-0,13$ & 0,19 & $-0,04$ & $-0,26^{*}$ & $-0,34^{* *}$ & $0,32^{* *}$ & $0,52^{* * *}$ \\
\hline Subgrupo 2 & $-0,48 * * *$ & $-0,28^{*}$ & $-0,05$ & $-0,06$ & $-0,52^{* * *}$ & $-0,38 * * *$ & $0,35^{* *}$ & $0,50 * * *$ \\
\hline Subgrupo 3 & $-0,17$ & $-0,29^{*}$ & $-0,21$ & $-0,40^{* *}$ & $-0,46^{* *}$ & $-0,35^{* *}$ & 0,19 & $0,51 * * *$ \\
\hline \multicolumn{9}{|l|}{ Grupo $C D A$} \\
\hline Subgrupo 1 & $-0,64^{* *}$ & $-0,71^{* * *}$ & $-0,23$ & $-0,31$ & $-0,47^{*}$ & $-0,47^{*}$ & 0,33 & 0,40 \\
\hline Subgrupo 2 & $-0,07$ & $-0,22$ & $-0,04$ & $-0,24$ & $-0,52^{*}$ & $-0,53^{*}$ & 0,42 & $0,48^{*}$ \\
\hline Subgrupo 3 & $-0,41$ & $-0,32$ & $-0,17$ & $-0,11$ & $-0,70 * *$ & $-0,45^{*}$ & 0,03 & $0,52^{*}$ \\
\hline
\end{tabular}

${ }^{*} p<0,05,{ }^{* *} p<0,01,{ }^{* * *} p<0,001$ 


\section{Regresiones Jerárquicas}

\section{Regresiones jerárquicas: grupo de niños SDA.}

Subgrupo 1. Los modelos completos de esta regresión jerárquica (variables del bloque 1 y 2 en conjunto) explicaron un 40,6\% $\left(R^{2}=0,406\right)$ de la varianza de la eficiencia en aritmética básica, $F(5,68)=9,28, p<0,001$. Las variables de control con contribución a la explicación de la varianza de la eficiencia en aritmética básica fueron edad, $B=-947,689, p=0,006,95 \%$ IC [-1610,839, -284,540], y eficiencia en comparación simbólica, $B=2,187, p=0,002,95 \%$ IC $[0,798,3,576]$. La eficiencia en comparación no-simbólica no mostró contribución única, $B=0,526, p=0,311$.

No hubo contribución única del puntaje en el span visuo-espacial progresivo en esta regresión jerárquica, $F(1,68)=0,06, B=7,144, p=0,808$, mientras que el puntaje obtenido en el span visuo-espacial regresivo mostró una contribución única del $3,5 \%\left(R^{2}=0,035\right)$ a la varianza de la eficiencia en aritmética básica en este grupo etario, $F(1,68)=4,02, B=-52,910, p=0,049,95 \%$ IC $[-105,600,-0,221]$. Ver detalles en la Tabla 3.

Tabla 3

Contribución Única de los Componentes de MT a la Varianza de la Eficiencia en Aritmética Básica en el Grupo de Niños SDA: Subgrupo 1

\begin{tabular}{lcccc}
\hline & $\boldsymbol{R}^{2}$ & $\Delta \boldsymbol{R}^{2}$ & $\Delta \boldsymbol{F}$ & Beta \\
\hline Modelo 1 & & & & \\
Edad & 0,405 & 0,405 & $11,746^{* * *}$ & $-0,277$ \\
Comparación no-simbólica & & & & 0,109 \\
Comparación simbólica & & & 0,059 & $-0,373$ \\
Span visuo-espacial regresivo & 0,406 & 0,001 & & 0,029 \\
Span visuo-espacial progresivo & & & & \\
Modelo 2 & 0,371 & 0,371 & & $-0,277$ \\
Edad & & & & 0,109 \\
Comparación no-simbólica & & & & 0,373 \\
Comparación simbólica & 0,406 & 0,035 & $4,015^{*}$ & $-0,229$ \\
Span visuo-espacial progresivo & & & \\
Span visuo-espacial regresivo & & & & \\
\hline
\end{tabular}

Nota. En todas las tablas que muestran los resultados de las regresiones jerárquicas (Tablas 3 a la 8) la primera columna a la izquierda representa el orden en que se introdujeron las variables en el modelo. Las siguientes columnas contienen los resultados de cada regresión: $R^{2}$ indica el porcentaje de variación de la variable dependiente explicada por el conjunto de variables independientes; $\Delta R^{2}$ indica la varianza única de la variable dependiente que es explicada por la variable independiente, controlando el efecto del resto de las variables independientes incluidas en el modelo; $\Delta F$ indica el cambio en la distribución de Fisher y tiene asociado un valor $p$ que indica si el valor $\Delta R^{2}$ es significativo o no. Beta se refiere al coeficiente Beta $(B)$ tipificado y ofrece una estimación del peso relativo que tiene cada variable independiente sobre la variable dependiente. El signo de este último coeficiente puede no ser el mismo que el coeficiente de correlación simple entre esta variable y la dependiente, debido a los ajustes que se llevan a cabo para la obtención de la mejor ecuación posible.

${ }^{*} p<0,05,{ }^{* * *} p<0,001$

Subgrupo 2. Los modelos completos de esta regresión jerárquica (variables del bloque 1 y 2 en conjunto) explicaron un 57\% $\left(R^{2}=0,570\right)$ de la varianza de la eficiencia en aritmética básica, $F(6,68)=15,02, p<0,001$. Las variables de control que contribuyeron a la explicación de la varianza de la eficiencia en aritmética básica fueron edad, $B=-799,013, p<0,001,95 \%$ IC [-1099,469, -498,556], eficiencia en comparación no-simbólica, $\mathrm{B}=0,572, p=0,027,95 \%$ IC $[0,066,1,078]$. La eficiencia en comparación simbólica, $B=0,645, p=0,47$, y el puntaje obtenido en el Raven, $B=11,717, p=0,783$, no mostraron contribución única.

No hubo contribución única del puntaje en el span visuo-espacial regresivo en esta regresión jerárquica, $F(1,68)=1,06, B=-14,965, p=0,307$, mientras que el puntaje obtenido en el span visuo-espacial progresivo mostró una contribución única del $3,2 \%\left(R^{2}=0,032\right)$ a la explicación de la varianza de la eficiencia en 
aritmética básica en este grupo etario, $F(1,68)=5,06, B=-33,217, p=0,028,95 \%$ IC [-62,692, -3,741]. Ver detalles en la Tabla 4.

Tabla 4

Contribución Única de los Componentes de MT a la Varianza de la Eficiencia en Aritmética

Básica en el Grupo de Niños SDA: Subgrupo 2

\begin{tabular}{lcccc}
\hline & $\boldsymbol{R}^{2}$ & $\Delta \boldsymbol{R}^{2}$ & $\Delta \boldsymbol{F}$ & Beta \\
\hline Modelo 1 & & & & \\
Edad & 0,538 & 0,538 & $16,063^{* * *}$ & $-0,463$ \\
Puntaje Raven & & & 0,025 \\
Comparación no-simbólica & & & & 0,228 \\
Comparación simbólica & & & $5,057^{*}$ & 0,212 \\
Span visuo-espacial regresivo & 0,570 & 0,032 & & $-0,108$ \\
Span visuo-espacial progresivo & & & $17,792^{* * *}$ & \\
Modelo 2 & 0,563 & 0,563 & & $-0,463$ \\
Edad & & & & 0,025 \\
Puntaje Raven & & & & 0,228 \\
Comparación no-simbólica & & & & 0,212 \\
Comparación simbólica & 0,570 & 0,007 & 1,057 & $-0,247$ \\
Span visuo-espacial progresivo & & & & \\
Span visuo-espacial regresivo & & & & \\
\hline
\end{tabular}

$* p<0,05,{ }^{* * *} p<0,001$

Subgrupo 3. Los modelos completos de esta regresión jerárquica (variables del bloque 1 y 2 en conjunto) explicaron un 49,8\% $\left(R^{2}=0,498\right)$ de la varianza de la eficiencia en aritmética básica, $F(5,69)=13,71$, $p<0,001$. Las variables de control que contribuyeron a la explicación de la varianza de la eficiencia en aritmética básica fueron puntaje en el Raven, $B=-28,237, p=0,020,95 \%$ IC [-51,900, -4,573], y la eficiencia en comparación simbólica, $B=1,555, p<0,001,95 \%$ IC [0,796, 2,313].

No hubo contribución única del puntaje en el span visuo-espacial regresivo en esta regresión jerárquica, $F(1,69)=0,39, B=-6,541, p=0,534$, mientras que el puntaje obtenido en el span visuo-espacial progresivo mostró una contribución única del $5,5 \%\left(R^{2}=0,055\right)$ a la explicación de la varianza de la eficiencia en aritmética básica en este grupo etario, $F(1,69)=7,50, B=-27,762, p=0,008,95 \%$ IC $[-47,980,-7,544]$. El puntaje obtenido en el span de dígitos regresivo también aportó una contribución única del $3,8 \%\left(R^{2}=0,038\right)$ a la varianza de la eficiencia en aritmética básica en este grupo etario, $F(1,69)=5,18, B=-128,072, p=0,026$, 95\% IC [-240,364, -15,779]. Ver detalles en la Tabla 5.

\section{Regresiones jerárquicas: grupo de niños CDA.}

Subgrupo 1. Los modelos completos de esta regresión jerárquica (variables del bloque 1 y 2 en conjunto) explicaron un 70,4\% $\left(R^{2}=0,704\right)$ de la varianza de la eficiencia en aritmética básica, $F(4,15)=8,91, p=0,001$. Las variables de control que contribuyeron a la explicación de la varianza de la eficiencia en aritmética básica fueron edad, $B=-2150,620, p=0,009$, 95\% IC [-3681,860, -619,380], y puntaje en el Raven, $B=-459,561$, $p=0,005,95 \%$ IC $[-760,363,-158,759]$.

No hubo contribución única del puntaje en el span visuo-espacial progresivo a la varianza de la eficiencia en aritmética básica en este grupo etario, $F(1,15)=0,46, B=75,703, p=0,509$. Tampoco la variable puntaje en el span visuo-espacial regresivo mostró una contribución única, $F(1,15)=0,26, B=-25,898, p=0,617$. Ver detalles en la Tabla 6.

Subgrupo 2. Los modelos completos de esta regresión jerárquica (variables del bloque 1 y 2 en conjunto) explicaron un $38,7 \%\left(R^{2}=0,387\right)$ de la varianza de la eficiencia en aritmética básica, $F(3,16)=3,36, p=0,045$. La variable de control eficiencia en comparación simbólica no mostró contribución única, $B=0,527, p=0,419$. 
Tabla 5

Contribución Única de los Componentes de MT a la Varianza de la Eficiencia en Aritmética Básica en el Grupo de Niños SDA: Subgrupo 3

\begin{tabular}{lcccc}
\hline & $\boldsymbol{R}^{2}$ & $\Delta \boldsymbol{R}^{2}$ & $\Delta \boldsymbol{F}$ & Beta \\
\hline Modelo 1 & & & & \\
Puntaje Raven & 0,444 & 0,444 & $13,961^{* * *}$ & $-0,206$ \\
$\begin{array}{l}\text { Comparación simbólica } \\
\text { Span de dígitos regresivo }\end{array}$ & & & 0,370 \\
Span visuo-espacial regresivo & & & & $-0,209$ \\
Span visuo-espacial progresivo & 0,498 & 0,055 & $7,504^{* *}$ & $-0,700$ \\
Modelo 2 & & & & $-0,306$ \\
Puntaje Raven & 0,495 & 0,495 & $17,186^{* * *}$ & $-0,206$ \\
Comparación simbólica & & & & $-0,370$ \\
Span de dígitos regresivo & & & & $-0,209$ \\
Span visuo-espacial progresivo & 0,498 & 0,003 & 0,391 & $-0,700$ \\
Span visuo-espacial regresivo & & & & $-0,206$ \\
Modelo 3 & 0,461 & 0,461 & & 0,370 \\
$\begin{array}{l}\text { Puntaje Raven } \\
\text { Comparación simbólica }\end{array}$ & & & & $-0,306$ \\
Span visuo-espacial progresivo & & & & $-0,700$ \\
Span visuo-espacial regresivo & & & & $-0,209$ \\
Span de dígitos regresivo & 0,498 & 0,038 & & \\
\hline
\end{tabular}

${ }^{*} p<0,05,{ }^{* *} p<0,01,{ }^{* * *} p<0,001$

Tabla 6

Contribución Única de los Componentes de MT a la Varianza de la Eficiencia en Aritmética Básica en el Grupo de Niños CDA: Subgrupo 1

\begin{tabular}{lcccc} 
& $\boldsymbol{R}^{2}$ & $\Delta \boldsymbol{R}^{2}$ & $\Delta \boldsymbol{F}$ & Beta \\
\hline $\begin{array}{l}\text { Modelo 1 } \\
\text { Edad }\end{array}$ & 0,695 & 0,695 & $12,138^{* * *}$ & $-0,516$ \\
$\begin{array}{l}\text { Puntaje Raven } \\
\text { Span visuo-espacial regresivo }\end{array}$ & & & & $-0,570$ \\
Span visuo-espacial progresivo & 0,704 & 0,009 & 0,458 & $-0,095$ \\
Modelo 2 & & & & 0,136 \\
Edad & 0,699 & 0,699 & $12,363^{* * * *}$ & $-0,516$ \\
$\begin{array}{l}\text { Puntaje Raven } \\
\text { Span visuo-espacial progresivo }\end{array}$ & & & & $-0,570$ \\
Span visuo-espacial regresivo & 0,704 & 0,005 & 0,261 & $-0,095$ \\
\hline
\end{tabular}

$* * * p<0,001$

No hubo contribución única del puntaje en el span visuo-espacial progresivo a la varianza de la eficiencia en aritmética básica en este grupo etario, $F(1,16)=0,92, B=-46,758, p=0,352$. Tampoco la variable puntaje en el span visuo-espacial regresivo mostró contribución única, $F(1,16)=1,67, B=-83,575, p=0,215$. Ver detalles en la Tabla 7.

Subgrupo 3. Los modelos completos de esta regresión jerárquica (variables del bloque 1 y 2 en conjunto) explicaron un 55,1\% $\left(R^{2}=0,551\right)$ de la varianza de la eficiencia en aritmética básica, $F(3,16)=6,54, p=0,004$. La variable de control eficiencia en comparación simbólica no mostró contribución única, $B=3,128, p=0,176$. 
Tabla 7

Contribución Única de los Componentes de MT a la Varianza de la Eficiencia en Aritmética Básica en el Grupo de Niños CDA: Subgrupo 2

\begin{tabular}{lcccc}
\hline & $\boldsymbol{R}^{2}$ & $\Delta \boldsymbol{R}^{2}$ & $\Delta \boldsymbol{F}$ & Beta \\
\hline $\begin{array}{l}\text { Modelo 1 } \\
\text { Comparación simbólica }\end{array}$ & 0,352 & 0,352 & $4,608^{*}$ & 0,200 \\
$\begin{array}{l}\text { Span visuo-espacial regresivo } \\
\text { Span visuo-espacial progresivo }\end{array}$ & 0,387 & 0,035 & 0,917 & $-0,310$ \\
Modelo 2 & & & & $-0,242$ \\
Comparación simbólica & 0,323 & 0,323 & $4,052^{*}$ & 0,200 \\
$\begin{array}{l}\text { Span visuo-espacial progresivo } \\
\text { Span visuo-espacial regresivo }\end{array}$ & 0,387 & 0,064 & & $-0,242$ \\
\hline * & & & & $-0,310$ \\
\hline
\end{tabular}

$* p<0,05$

No hubo contribución única del puntaje en el span visuo-espacial progresivo a la varianza de la eficiencia en aritmética básica en este grupo etario, $F(1,16)=3,46, B=-195,650, p=0,081$. Tampoco la variable puntaje en el span visuo-espacial regresivo mostró contribución única, $F(1,16)=0,21, B=-32,039, p=0,656$. Ver detalles en la Tabla 8.

Tabla 8

Contribución Única de los Componentes de MT a la Varianza de la Eficiencia en Aritmética Básica en el Grupo de Niños CDA: Subgrupo 3

\begin{tabular}{lcccc}
\hline & $\boldsymbol{R}^{2}$ & $\Delta \boldsymbol{R}^{2}$ & $\Delta \boldsymbol{F}$ & Beta \\
\hline $\begin{array}{l}\text { Modelo 1 } \\
\text { Comparación simbólica }\end{array}$ & 0,453 & 0,453 & $7,053^{* *}$ & 0,289 \\
$\begin{array}{l}\text { Span visuo-espacial regresivo } \\
\text { Span visuo-espacial progresivo }\end{array}$ & 0,551 & 0,097 & 3,458 & $-0,108$ \\
$\begin{array}{l}\text { Modelo 2 } \\
\text { Comparación simbólica }\end{array}$ & 0,545 & 0,545 & $10,175^{* *}$ & $-0,499$ \\
$\begin{array}{l}\text { Span visuo-espacial progresivo } \\
\text { Span visuo-espacial regresivo }\end{array}$ & 0,551 & 0,006 & 0,206 & $-0,499$ \\
\hline
\end{tabular}

$* * p<0,01$

\section{Comparaciones entre los Grupos SDA y CDA en las Tareas que Evaluaron los Componentes de la MT}

A diferencia de los resultados encontrados en el grupo SDA, en el grupo de niños CDA ninguno de los componentes de la MT mostró una contribución única que explique la varianza de la eficiencia en aritmética básica. Por esta razón, se realizaron ANOVAs de medidas repetidas para explorar posibles diferencias entre los grupos en el rendimiento en las tareas que exploraron MT. Para cada subgrupo (1, 2 y 3 ) se realizaron dos ANOVAs, uno con el puntaje obtenido en las tareas de MT verbal (span de dígitos progresivo y regresivo) y otro con el puntaje obtenido en las tareas de MT visuo-espacial (span visuo-espacial progresivo y regresivo). En ambos ANOVAs se incluyeron las medidas de MT como factores intrasujeto y el grupo (SDA, CDA) como factor intergrupo.

Subgrupo 1. Los resultados del análisis de las puntuaciones de MT verbal mostraron efecto del grupo, $F(1,93)=10,20, p=0,002, \eta_{p}^{2}=0,099,95 \%$ IC $[3,569,3,924]$ y $[2,781,3,468]$ para los grupos SDA y CDA, respectivamente, mostrando un mejor rendimiento el grupo SDA (Figura 1a). También se encontró efecto de la tarea, $F(1,93)=23,42, p<0,001, \mathrm{n}_{\mathrm{p}}^{2}=0,20,95 \%$ IC $[3,595,4,128]$ y $[2,755,3,264]$ para span de dígitos 
progresivo y regresivo, respectivamente. Es así que las comparaciones planeadas arrojaron diferencias entre los grupos, tanto en el span de dígitos progresivo, $p=0,022$, 95\% IC [3,929, 4,418] y [3,077, 4,023], como en el span de dígitos regresivo, $p=0,017,95 \%$ IC [3,086,3,553] y [2,247, 3,152]. Los intervalos de confianza en ambos casos corresponden a los grupos SDA y CDA, respectivamente. No se encontró interacción entre grupo $\mathrm{y}$ tareas de MT, $F(1,93)=0,00, p=0,992$.

Los resultados del análisis de las puntuaciones de MT visuo-espacial también mostraron efecto del grupo, $F(1,93)=13,49, p<0,001, \eta_{\mathrm{p}}^{2}=0,127,95 \%$ IC $[20,621,24,165]$ y $[11,818,18,682]$ para los grupos SDA y CDA, respectivamente, con un mejor rendimiento en el grupo SDA (Figura 2a). Por su parte, no se encontró efecto de la tarea, $F(1,93)=3,18, p=0,178$.. Sin embargo, las comparaciones planeadas arrojaron diferencias entre los grupos, tanto en el span visuo-espacial progresivo, $p<0,001,95 \%$ IC $[19,920,23,706]$ y $[10,134$, $17,466]$, como en el span visuo-espacial regresivo, $p=0,011,95 \%$ IC $[20,771,25,176]$ y $[12,435,20,965]$. Los intervalos de confianza en ambos casos corresponden a los grupos SDA y CDA, respectivamente. No se encontró interacción entre grupo y tareas de MT, $F(1,93)=0,58, p=0,447$.

Subgrupo 2. Los resultados del análisis de las puntuaciones de MT verbal mostraron un efecto del grupo, $F(1,93)=4,25, p=0,042, \eta_{\mathrm{p}}^{2}=0,044,95 \%$ IC $[4,160,4,613]$ y $[3,462,4,338]$ para los grupos SDA y CDA, respectivamente. También se encontró efecto de la tarea, $F(1,93)=17,80, p<0,001, \mathrm{\eta}_{\mathrm{p}}{ }^{2}=0,16,95 \% \mathrm{IC}[4,198$, $4,862]$ y [3,470, 4,043] para span de dígitos progresivo y regresivo, respectivamente. Pese a ello, las comparaciones planeadas no mostraron diferencias entre los grupos en ninguna de las dos tareas (span de dígitos progresivo: $p=0,130$ y regresivo: $p=0,078)$. Ver Figura 1b. No se encontró interacción entre grupo y tareas de MT, $F(1,93)=-0,00, p>0,999$.

Los resultados del análisis de las puntuaciones de MT visuo-espacial también mostraron un efecto del grupo, $F(1,93)=4,66, p=0,033, \eta_{\mathrm{p}}^{2}=0,048,95 \%$ IC [30,378, 33,995] y [24,398, 31,402] para los grupos SDA y CDA, respectivamente, mostrando un mejor rendimiento el grupo SDA (Figura 2b). No obstante, no se encontró efecto de la tarea, $F(1,93)=0,53, p=0,467$. Sin embargo, las comparaciones planeadas arrojaron diferencias entre los grupos en el span visuo-espacial progresivo, $p=0,031,95 \%$ IC $[19,920,23,706]$ y $[10,134$, 17,466] para los grupos SDA y CDA, respectivamente. No se encontró interacción entre grupo y tareas de MT, $F(1,93)=0,61, p=0,435$.

Subgrupo 3. Los resultados del análisis de las puntuaciones de MT verbal no mostraron un efecto del grupo, $F(1,93)=1,14, p=0,289$, pero sí de la tarea, $F(1,93)=35,14, p<0,001, \mathrm{n}^{2}=0,27,95 \%$ IC $[4,825$, $5,609]$ y $[3,558,4,235]$ para span de dígitos progresivo y regresivo, respectivamente. Sin embargo, las comparaciones planeadas arrojaron diferencias entre los grupos en el span de dígitos regresivo, $p=0,022$, $95 \%$ IC $[3,983,4,604]$ y $[2,899,4,101]$ para los grupos SDA y CDA, respectivamente. Ver Figura 1c. También se encontró interacción entre grupo y tareas de MT, $F(1,93)=4,65, p=0,034, \mathrm{np}^{2}=0,05$ : mientras no se aprecia diferencia entre los grupos en el span de dígitos progresivo, en el de dígitos regresivo es el grupo SDA el que aventaja al grupo CDA.

A diferencia de las puntuaciones de MT verbal, los resultados del análisis de las puntuaciones de MT visuo-espacial sí mostraron un efecto del grupo, $F(1,93)=14,80, p<0,001, \eta_{\mathrm{p}}^{2}=0,14,95 \%$ IC $[33,804,37,623]$ y $[23,952,31,348]$ para los grupos SDA y CDA, respectivamente. No se encontró efecto de la tarea, $F(1,93)=0,03, p=0,857$. Sin embargo, las comparaciones planeadas arrojaron diferencias entre los grupos, tanto en el span visuo-espacial progresivo, $p=0,001,95 \%$ IC [33,244, 37,422] y [23,804, 31,895], como en el span visuo-espacial regresivo, $p<0,001,95 \%$ IC [33,955, 38,231] y [23,310, 31,590]. Los intervalos de confianza en ambos casos corresponden a los grupos SDA y CDA, respectivamente. Ver Figura 2c. En esta tarea no se encontró interacción entre grupo y tareas de MT, $F(1,93)=0,34, p=0,561$. 


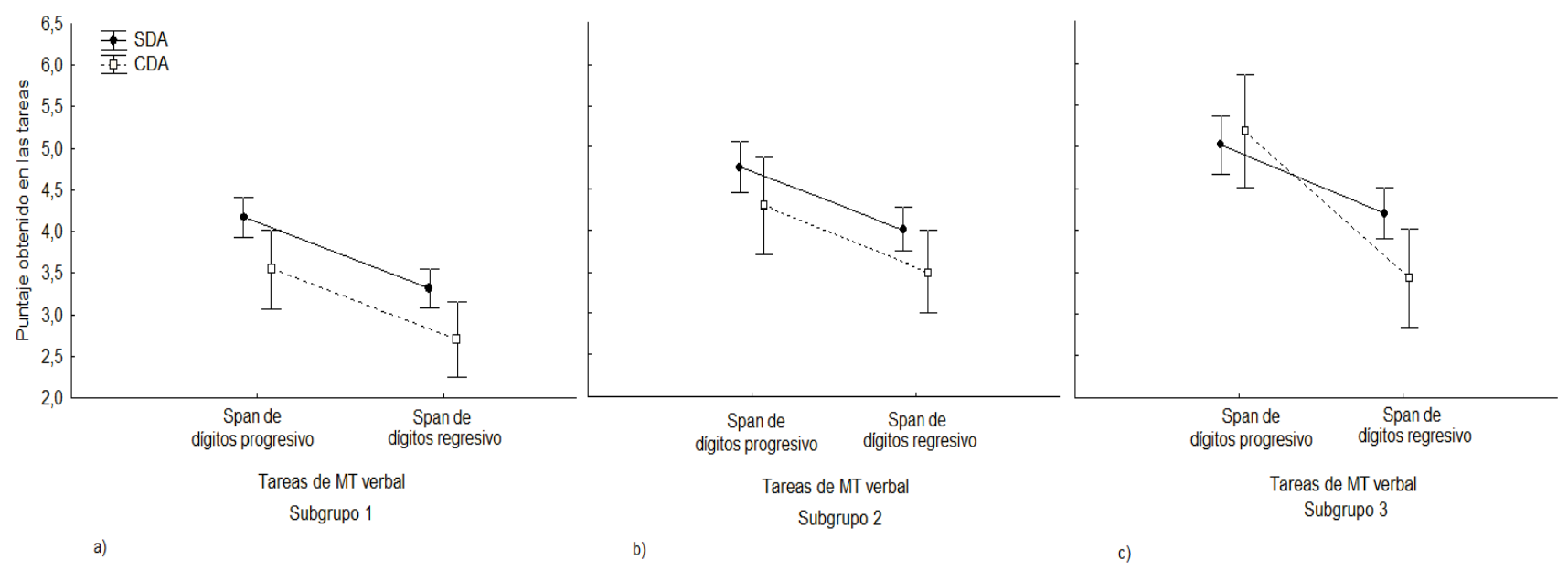

Figura 1. Resultados de las tareas de MT verbal por grupo (SDA versus CDA) para cada momento del desarrollo evaluado.

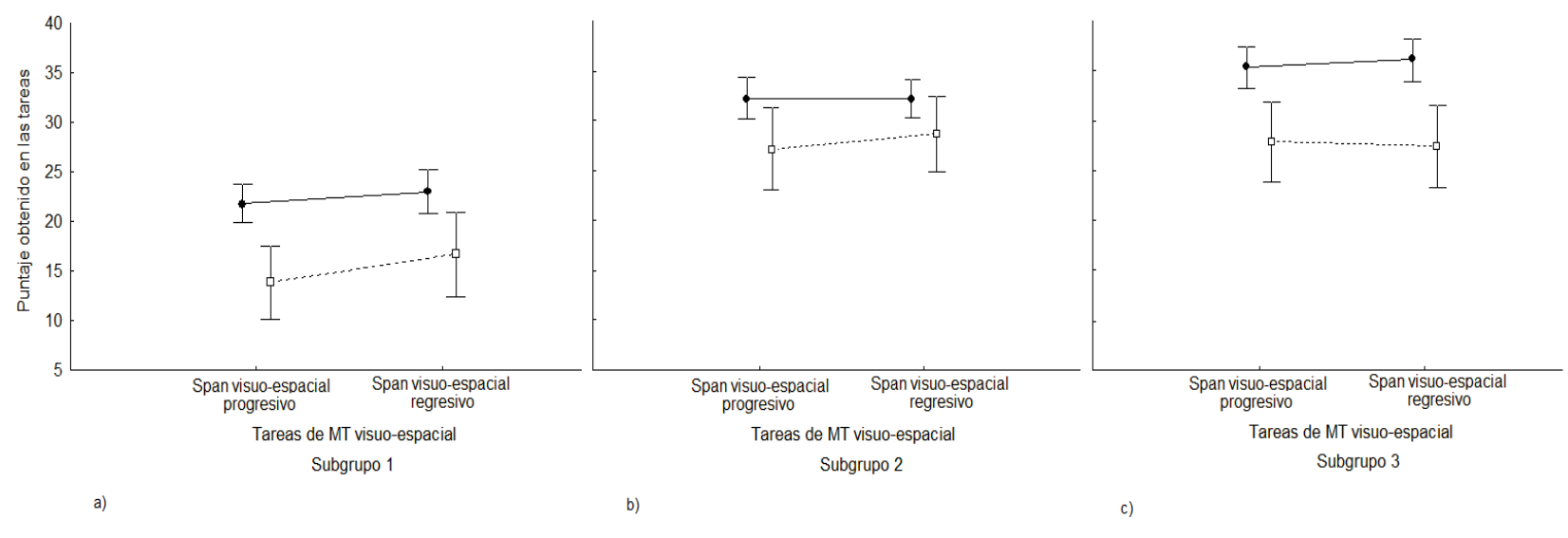

Figura 2. Resultados de las tareas de MT visuo-espacial por grupo (SDA versus CDA) para cada momento del desarrollo evaluado.

\section{Discusión}

Los resultados de este estudio mostraron que, aun controlando variables de dominio general y de dominio numérico, los componentes de la MT tienen una contribución única en la varianza de la eficiencia en aritmética básica, pero esta contribución varía en la medida que aumentan la edad y la exposición a la enseñanza formal de las matemáticas. Se encontró que en los niños SDA de menor edad ( $1^{\circ}$ y $2^{\circ}$ grados) solo el funcionamiento del componente ejecutivo central al procesar información visuo-espacial (span visuoespacial regresivo) mostró una contribución única en la explicación de la varianza en el rendimiento en aritmética básica, mientras que en los niños algo mayores $\left(3^{\circ}\right.$ y $4^{\circ}$ grados) solo la agenda visuo-espacial (span visuo-espacial progresivo) fue el componente con contribución única. Este componente también mostró una contribución única a la explicación de la varianza de la eficiencia en aritmética en los niños de mayor edad $\left(5^{\circ}\right.$ y $6^{\circ}$ grados), pero además otro porcentaje de dicha varianza estuvo explicado por el funcionamiento del componente ejecutivo central cuando se procesó información verbal (span de dígitos regresivo).

Estos resultados indican que, durante las etapas iniciales de exposición a los símbolos numéricos, cuando todavía no se ha consolidado el mapeo entre las cantidades no-simbólicas y sus etiquetas verbales, los niños, para realizar cálculos aritméticos, probablemente intenten primero ubicar espacialmente los dígitos en su línea numérica mental y luego utilicen procesos de conteo para obtener el resultado de la operación que 
requiere la tarea. Este tipo de estrategias demandaría la participación activa del componente ejecutivo central en su función de control atencional. Estudios previos han descrito que el desempeño aritmético de los niños de 6-8 años se ve afectado por dificultades en los componentes de la agenda visuo-espacial y ejecutivo central cuando se requiere del procesamiento de información visuo-espacial (Berg, 2008; Holmes \& Adams, 2006; McKenzie et al., 2003).

Los datos indican que ya alrededor de los 10 años el rendimiento en tareas aritméticas comienza a verse afectado no solo por los componentes visuo-espaciales de la MT, sino también por el procesamiento de la información verbal en el ejecutivo central. Estos resultados están muy vinculados a las características del desarrollo numérico y son similares a los resultados obtenidos por Holmes y Adams (2006) y McKenzie et al. (2003). Como se ha descrito anteriormente, en los primeros grados escolares el procesamiento numérico está muy ligado a las representaciones mentales no-simbólicas (por consiguiente, la aritmética aún depende mucho de las propiedades perceptuales de los estímulos) y es más trabajoso el acceso a las cantidades a través de los símbolos numéricos recientemente adquiridos. Sin embargo, con el avance escolar esta situación se invierte y se desarrolla cada vez más el mapeo entre los símbolos numéricos y las cantidades que estos representan. De esta forma, el niño comienza a sustituir las operaciones con objetos por operaciones con sistemas numéricos abstractos, los cuales son cada vez más dependientes de códigos verbales (Castro Cañizares, Estévez Pérez \& Pérez Marrero, 2011). Alrededor de los 10 años, usualmente los niños ya realizan las tareas de aritmética básica a través de la recuperación de hechos numéricos directamente desde la memoria a largo plazo, lo cual involucra al bucle fonológico (Dehaene \& Cohen, 1995) y refleja el dominio de la aritmética simbólico-lingüística basada en códigos verbales (Berg, 2008; Dehaene \& Cohen, 1995; Houdé, 1997). Lo anterior podría explicar el hecho de que hacia el final de la enseñanza escolar elemental $\left(5^{\circ}-6^{\circ}\right.$ grados), producto de la interiorización de los símbolos numéricos y de la automatización de las operaciones de suma y resta como hechos numéricos, el componente ejecutivo central de la MT (que incluye el almacenamiento realizado por el bucle fonológico y el procesamiento simultáneo de información verbal) tiene una mayor contribución única a la varianza de la eficiencia en aritmética.

$\mathrm{Al}$ analizar los resultados de los niños del grupo con dificultades en aritmética se encontró que los componentes visuo-espaciales de la MT se correlacionaron con la aritmética básica a lo largo de todo el desarrollo escolar, aunque, a diferencia del grupo SDA, estos componentes de la MT no mostraron contribución única a la explicación de la varianza de la eficiencia en aritmética básica de estos niños. Asimismo se encontró que el rendimiento del grupo CDA en las tareas de MT fue menor que el rendimiento del grupo SDA, particularmente en aquellos componentes que mostraron contribución única a la varianza de la eficiencia en aritmética básica en este último grupo. En su conjunto, estos resultados sugieren que una menor eficiencia de los procesos vinculados a la MT (principalmente a los componentes visuo-espaciales) podría estar provocando que estos niños sean menos eficientes en el mantenimiento de imágenes mentales vinculadas al espacio problema de las operaciones aritméticas y, por tanto, presenten dificultades en este dominio. Además, una menor operatividad de la MT también podría interferir en la calidad de los procesos de codificación y recuperación de los hechos numéricos, procesos descritos como muy vinculados con la MT verbal (Dehaene \& Cohen, 1995; Fuchs et al., 2006). En general, los resultados de este grupo de niños CDA sustentan el análisis de Szucs et al. (2013), quienes proponen, después de un análisis de varias investigaciones conductuales y de imágenes cerebrales, que las dificultades que aparecen durante el aprendizaje de las matemáticas (y particularmente en la discalculia del desarrollo) se deben fundamentalmente a una disfunción en la MT visuo-espacial vinculada al funcionamiento del ejecutivo central en la MT, más que a una disfunción en el procesamiento de las magnitudes. Lo anterior lleva a considerar que, aunque las habilidades numéricas básicas también explican un porcentaje de la varianza de la eficiencia en aritmética, el origen las dificultades en el aprendizaje de las matemáticas no parece radicar solo en un déficit en el desarrollo de habilidades numéricas básicas o en el mapeo entre los símbolos numéricos y las cantidades que estos representan, como señalan las teorías actuales sobre el origen de los trastornos en el desarrollo numérico (Dehaene, 1997; Rousselle \& Noël, 2007), sino que el funcionamiento de los componentes de la MT también puede estar muy involucrado en la aparición de las dificultades en el aprendizaje de las matemáticas.

De manera general, en este estudio se encontró que los componentes de la MT tienen una contribución única en la explicación de la varianza de la eficiencia en la aritmética básica durante la edad escolar. Estos datos apoyan resultados previos (Fuchs et al., 2010; Swanson, 2011) y aportan nuevas evidencias respecto a cuáles componentes de la MT contribuyen de manera específica al rendimiento aritmético básico en cada momento del desarrollo escolar (aun cuando se controlen variables de dominio general y específico). Lo 
anterior resulta de utilidad en la descripción de cómo se produce el aprendizaje de las matemáticas durante la edad escolar. En consecuencia, sería oportuno considerar la MT como componente en los modelos teóricos para explicar el desarrollo de la cognición numérica. La MT debería ser una variable a considerar tanto en la identificación de niños en riesgo de presentar dificultades en las matemáticas como en los análisis de predictores del desempeño matemático posterior.

Respecto de la práctica educativa, dado que los componentes visuales de la MT están muy vinculados al rendimiento aritmético durante los primeros años escolares, los docentes podrían enfatizar métodos de enseñanza apoyados principalmente en las características visuo-espaciales del contenido numérico a enseñar (e.g., el uso de pancartas con instrucciones visibles todo el tiempo, uso de procedimientos enumerados para la resolución de ejercicios), hasta que los niños comiencen a automatizar el uso de los símbolos numéricos y aumenten, así, su competencia en el uso de estrategias verbales abstractas.

En último lugar, algunos elementos relacionados con el diseño de este estudio deben ser comentados y considerados en futuras investigaciones. Uno de estos elementos es el uso de la eficiencia en cálculo básico exacto (con números de un solo dígito) como variable para evaluar el rendimiento aritmético. Como se describió anteriormente, el rendimiento en este tipo de tareas es considerado como predictor del posterior desempeño matemático. Sin embargo, la rápida automatización que tienen las adiciones y sustracciones simples, convirtiéndose en hechos numéricos recuperables desde la memoria a corto plazo, podría no abarcar la implicación real de las funciones ejecutivas en el procesamiento aritmético. En este sentido, en estudios futuros se deberían incluir tareas de cálculo con números mayores y tareas de cálculos aproximados, ya que el rol de los procesos de memoria podría diferenciarse ante diferentes tipos de cálculos. Por otro lado, las tareas utilizadas en este estudio para la evaluación del componente ejecutivo central de la MT solo permitieron explorar la función de almacenamiento y procesamiento simultáneo. Para una evaluación más exhaustiva de este componente sería necesario incluir tareas N-back y/o tareas duales que permitiesen evaluar otras funciones, como la actualización de contenidos en la MT, la flexibilidad cognitiva y el control inhibitorio (Bull \& Lee, 2014; Cragg \& Gilmore, 2014). Asimismo, al considerar las variables de dominio específico que podrían explicar la varianza en la eficiencia en la aritmética, podrían incluirse otras que también se han descrito como predictores del desempeño en matemáticas. Tal es el caso del conteo y de la estimación instantánea de las cantidades menores que cuatro.

\section{Referencias}

Alloway, T. P., Gathercole, S. E., Adams, A. -M., Willis, C., Eaglen, R. \& Lamont, E. (2005). Working memory and phonological awareness as predictors of progress towards early learning goals at school entry. British Journal of Developmental Psychology, 23, 417-426. https://doi.org/10.1348/026151005X26804

Amor Díaz, V. \& Torres Díaz, R. (2016). Memoria de trabajo, inteligencia fluida y rendimiento académico en niños de edad escolar. Revista Neuropsicología, Neuropsiquiatría y Neurociencias, 16(3), 15-33.

Baddeley, A. (1992). Is working memory working? The fifteenth Bartlett lecture. The Quarterly Journal of Experimental Psychology, 44, 1-31. https://doi.org/10.1080/14640749208401281

Baddeley, A. (1996). The fractionation of working memory. Proceedings of the National Academy of Sciences, 93, $13468-13472$. https://doi.org/10.1073/pnas.93.24.13468

Baddeley, A. (2003). Working memory: Looking back and looking forward. Nature Reviews Neuroscience, 4, 829-839. https://doi.org/10.1038/nrn1201

Baddeley, A. D. \& Hitch, G. (1974). Working memory. Psychology of Learning and Motivation, 8, 47-89. https://doi.org/10.1016/S0079$7421(08) 60452-1$

Baddeley, A. D. \& Hitch, G. J. (1994). Developments in the concept of working memory. Neuropsychology, 8, 485-493. https://doi.org/10.1037/0894-4105.8.4.485

Berg, D. H. (2008). Working memory and arithmetic calculation in children: The contributory roles of processing speed, short-term memory, and reading. Journal of Experimental Child Psychology, 99, 288-308. https://doi.org/10.1016/j.jecp.2007.12.002

Bull, R. \& Johnston, R. S. (1997). Children's arithmetical difficulties: Contributions from processing speed, item identification, and short-term memory. Journal of Experimental Child Psychology, 65, 1-24. https://doi.org/10.1006/jecp.1996.2358

Bull, R. \& Lee, K. (2014). Executive functioning and mathematics achievement. Child Development Perspectives, 8, 36-41. https://doi.org/10.1111/cdep.12059

Castro Cañizares, D., Estévez Pérez, N. \& Pérez Marrero, O. (2011). Typical development of quantity comparison in school-aged children. The Spanish Journal of Psychology, 14, 50-61. https://doi.org/10.5209/rev_SJOP.2011.v14.n1.4

Cornoldi, C., Rigoni, F., Venneri, A. \& Vecchi, T. (2000). Passive and active processes in visuo-spatial memory: Double dissociation in developmental learning disabilities. Brain and Cognition, 43, 117-120.

Cragg, L. \& Gilmore, C. (2014). Skills underlying mathematics: The role of executive function in the development of mathematics proficiency. Trends in Neuroscience and Education, 3, 63-68. https://doi.org/10.1016/j.tine.2013.12.001

Crawford, J. R. \& Howell, D. C. (1998). Comparing an individual's test score against norms derived from small samples. The Clinical Neuropsychologist, 12, 482-486. https://doi.org/10.1076/clin.12.4.482.7241 
David, C. V. (2012). Working memory deficits in math learning difficulties: A meta-analysis. International Journal of Developmental Disabilities, 58, 67-84. https://doi.org/10.1179/2047387711Y.0000000007

Dehaene, S. (1992). Varieties of numerical abilities. Cognition, 44, 1-42. https://doi.org/10.1016/0010-0277(92)90049-N

Dehaene, S. (1997). The number sense: How the mind creates mathematics. New York, NY: Oxford University Press.

Dehaene, S. \& Cohen, L. (1995). Towards an anatomical and functional model of number processing. Mathematical Cognition, 1, 83-120.

Dehaene, S., Piazza, M., Pinel, P. \& Cohen, L. (2003). Three parietal circuits for number processing. Cognitive Neuropsychology, 20, 487-506. https://doi.org/10.1080/02643290244000239

De Smedt, B., Verschaffel, L. \& Ghesquière, P. (2009). The predictive value of numerical magnitude comparison for individual differences in mathematics achievement. Journal of Experimental Child Psychology, 103, 469-479. https://doi.org/10.1016/j.jecp.2009.01.010

Dumontheil, I. \& Klingberg, T. (2012). Brain activity during a visuospatial working memory task predicts arithmetical performance 2 years later. Cerebral Cortex, 22, 1078-1085. https://doi.org/10.1093/cercor/bhr175

Feigenson, L., Dehaene, S. \& Spelke, E. (2004). Core systems of number. Trends in Cognitive Sciences, 8, 307-314. https://doi.org/10.1016/j.tics.2004.05.002

Friso-van den Bos, I., van der Ven, S. H. G., Kroesbergen, E. H. \& van Luit, J. E. H. (2013). Working memory and mathematics in primary school children: A meta-analysis. Educational Research Review, 10, 29-44. https://doi.org/10.1016/j.edurev.2013.05.003

Fuchs, L. S., Fuchs, D., Compton, D. L., Powell, S. R., Seethaler, P. M., Capizzi, A. M. ... Fletcher, J. M. (2006). The cognitive correlates of third-grade skill in arithmetic, algorithmic computation, and arithmetic word problems. Journal of Educational Psychology, 98, 29-43. https://doi.org/10.1037/0022-0663.98.1.29

Fuchs, L. S., Geary, D. C., Compton, D. L., Fuchs, D., Hamlett, C. L. \& Bryant, J. D. (2010). The contributions of numerosity and domaingeneral abilities to school readiness. Child Development, 81, 1520-1533. https://doi.org/10.1111/j.1467-8624.2010.01489.x

Geary, D. C. (2011). Consequences, characteristics, and causes of mathematical learning disabilities and persistent low achievement in mathematics. Journal of Developmental \& Behavioral Pediatrics, 32, 250-263. https://doi.org/10.1097/DBP.0b013e318209edef

Holmes, J. \& Adams, J. W. (2006). Working memory and children's mathematical skills: Implications for mathematical development and mathematics curricula. Educational Psychology, 26, 339-366. https://doi.org/10.1080/01443410500341056

Houdé, O. (1997). Numerical development: From the infant to the child. Wynn's (1992) paradigm in 2- and 3-year olds. Cognitive Development, 12, 373-391. https://doi.org/10.1016/S0885-2014(97)90009-5

Hyde, D. C., Khanum, S. \& Spelke, E. S. (2014). Brief non-symbolic, approximate number practice enhances subsequent exact symbolic arithmetic in children. Cognition, 131, 92-107. https://doi.org/10.1016/j.cognition.2013.12.007

Jordan, N. C. \& Montani, T. O. (1997). Cognitive arithmetic and problem solving: A comparison of children with specific and general mathematics difficulties. Journal of Learning Disabilities, 30, 624-634. https://doi.org/10.1177/002221949703000606

Kyttälä, M. \& Lehto, J. E. (2008). Some factors underlying mathematical performance: The role of visuospatial working memory and non-verbal intelligence. European Journal of Psychology of Education, 23, 77-94. https://doi.org/10.1007/BF03173141

LeFevre, J. -A., Fast, L., Skwarchuk, S. -L., Smith-Chant, B. L., Bisanz, J., Kamawar, D. \& Penner-Wilger, M. (2010). Pathways to mathematics: Longitudinal predictors of performance. Child Development, 81, 1753-1767. https://doi.org/10.1111/j.14678624.2010.01508.x

Logie, R. H. (1995). Visuo-spatial working memory. Hove, Reino Unido: Psychology Press.

McCloskey, M. (1992). Cognitive mechanisms in numerical processing: Evidence from acquired dyscalculia. Cognition, 44, 107-157. https://doi.org/10.1016/0010-0277(92)90052-J

McKenzie, B., Bull, R. \& Gray, C. (2003). The effects of phonological and visual-spatial interference on children's arithmetical performance. Educational \& Child Psychology, 20(3), 93-108.

McLean, J. F. \& Hitch, G. J. (1999). Working memory impairments in children with specific arithmetic learning difficulties. Journal of Experimental Child Psychology, 74, 240-260. https://doi.org/10.1006/jecp.1999.2516

Passolunghi, M. C. \& Cornoldi, C. (2008). Working memory failures in children with arithmetical difficulties. Child Neuropsychology, 14, 387-400. https://doi.org/10.1080/09297040701566662

Passolunghi, M. C. \& Siegel, L. S. (2001). Short-term memory, working memory, and inhibitory control in children with difficulties in arithmetic problem solving. Journal of Experimental Child Psychology, 80, 44-57. https://doi.org/10.1006/jecp.2000.2626

Passolunghi, M. C. \& Siegel, L. S. (2004). Working memory and access to numerical information in children with disability in mathematics. Journal of Experimental Child Psychology, 88, 348-367. https://doi.org/10.1016/j.jecp.2004.04.002

Quinn, J. G. \& McConnell, J. (1999). Manipulation of interference in the passive visual store. European Journal of Cognitive Psychology, 11, 373-390. https://doi.org/10.1080/713752322

Raven, J. C., Court, J. H. \& Raven, J. (1992). Manual for Raven's progressive matrices and vocabulary scales. Oxford, Reino Unido: Oxford Psychologists Press.

Reigosa-Crespo, V., González-Alemañy, E., León, T., Torres, R., Mosquera, R. \& Valdés-Sosa, M. (2013). Numerical capacities as domainspecific predictors beyond early mathematics learning: A longitudinal study. PloS One, 8(11), artículo e79711. https://doi.org/10.1371/journal.pone.0079711

Rousselle, L. \& Noël, M. -P. (2007). Basic numerical skills in children with mathematics learning disabilities: A comparison of symbolic vs non-symbolic number magnitude processing. Cognition, 102, 361-395. https://doi.org/10.1016/j.cognition.2006.01.005

Sattler, J. M. (1982). Assessment of children's intelligence and special abilities (2a ed.). Boston, MA: Allyn \& Bacon.

Swanson, H. L. (2011). Working memory, attention, and mathematical problem solving: A longitudinal study of elementary school children. Journal of Educational Psychology, 103, 821-837. https://doi.org/10.1037/a0025114

Swanson, L. \& Jerman, O. (2006). Math disabilities: A preliminary meta-analysis of the published literature on cognitive processes. En T. E. Scruggs \& M. A. Mastropieri (Eds.), Applications of research methodology (pp. 285-314). Oxford, Reino Unido: JAI Press.

Szucs, D., Devine, A., Soltesz, F., Nobes, A. \& Gabriel, F. (2013). Developmental dyscalculia is related to visuo-spatial memory and inhibition impairment. Cortex, 49, 2674-2688. https://doi.org/10.1016/j.cortex.2013.06.007

Tillman, C. M., Nyberg, L. \& Bohlin, G. (2008). Working memory components and intelligence in children. Intelligence, 36, $394-402$. https://doi.org/10.1016/j.intell.2007.10.001

Träff, U. (2013). The contribution of general cognitive abilities and number abilities to different aspects of mathematics in children. Journal of Experimental Child Psychology, 116, 139-156. https://doi.org/10.1016/j.jecp.2013.04.007

Vecchi, T. \& Cornoldi, C. (1999). Passive storage and active manipulation in visuo-spatial working memory: Further evidence from the study of age differences. European Journal of Cognitive Psychology, 11, 391-406. https://doi.org/10.1080/713752324 
Wu, S. S., Meyer, M. L., Maeda, U., Salimpoor, V., Tomiyama, S., Geary, D. C. \& Menon, V. (2008). Standardized assessment of strategy use and working memory in early mental arithmetic performance. Developmental Neuropsychology, 33, 365-393. https://doi.org/10.1080/87565640801982445

Fecha de recepción: Diciembre de 2016.

Fecha de aceptación: Agosto de 2017. 